\title{
Life Inside an Acorn: How Microclimate and Microbes Influence Nest Organization in Temnothorax Ants
}

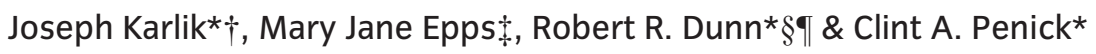 \\ * Department of Applied Ecology, North Carolina State University, Raleigh, NC, USA \\ $\uparrow$ Department of Physics and Astronomy, University of North Carolina, Chapel Hill, NC, USA \\ * Department of Biology, Mary Baldwin College, Staunton, VA, USA \\ $\S$ Keck Center for Behavioral Biology, North Carolina State University, Raleigh, NC, USA \\ - Center for Macroecology, Evolution and Climate, Natural History Museum of Denmark, University of Copenhagen, Copenhagen, Denmark
}

\section{Correspondence}

Clint A. Penick, Department of Applied Ecology, North Carolina State University, Raleigh, NC 27695-7617, USA.

E-mail: capenick@ncsu.edu

Received: March 13, 2016

Initial acceptance: April 21, 2016

Final acceptance: July 2, 2016

(S. Foster)

doi: $10.1111 /$ eth. 12525

Keywords: nest architecture, thermoregulation, microbes, pathogens, ants, Temnothorax

\begin{abstract}
Nests provide a buffer against environmental variation, but conditions may also vary at different locations within a nest. Conditions can vary based on abiotic factors, such as moisture and temperature, as well as biotic factors, such as the presence of microbes and potential pathogens. Therefore, characterizing how animals adjust their position inside their nests to track microclimate preferences while at the same time preventing pathogen exposure is necessary to understand the benefits nests provide. Here we studied how colonies of the acorn-nesting ant Temnothorax curvispinosus responded to experimental manipulation of moisture, temperature, and microbial growth inside their nests. Colonies showed no response to differences in moisture and moved to the bottom of the acorn regardless of moisture treatment. When nests were heated from the top to simulate warming by the sun, workers preferentially moved brood to the warm, upper half of the acorn, which would stimulate brood development. Finally, the strongest factor that influenced colony position was the presence of microbes inside the nest - colonies avoided the bottom of the nest when it was inoculated with microbes, and colonies in new acorns shifted to the top of the acorn over time as mold and other microbes had time to grow. The relatively strong response of $T$. curvispinosus to microbial growth inside their nests suggests that pathogen pressures - in addition to microclimate-have a significant impact on how colonies use nest spaces. Social insects are known to invest heavily in antimicrobial compounds that kill or slow the growth of microbes, but avoidance may represent an additional line of defense to prevent pathogen exposure.
\end{abstract}

\section{Introduction}

Nests are an extension of an animal's physiology that help shape the environment to which an animal is exposed (Turner 2009). Simple nests, such as tree cavities or underground burrows, provide shelter from extreme temperatures and a refuge against desiccation (Schwarzkopf \& Alford 1996; Kinlaw 1999; Wiebe 2001). More complex nests, such as those built by some ants and termites, feature chimneys and ventilation systems that maintain conditions as close as possible to a species' optimum (Korb 2003; Jones \&
Oldroyd 2006). Yet it is not only the structure of nests that determines their utility, but also how animals adjust their behavior to use these structures once they are inhabited. This is particularly true for ants and other social insects, whose colonies exhibit narrow temperature and moisture preferences (Potts et al. 1984; Roces \& Núñez 1989; Porter \& Tschinkel 1993; Mezger $\&$ Pfeiffer 2010). Colonies must adjust their position to track microclimate change while also limiting their exposure to microbes and potential pathogens that can proliferate inside nest spaces. How social insects are able to track their microclimate preferences 
while at the same time avoiding pathogen exposure will determine the true benefits nests provide.

Variation in nest temperature and moisture can have major impacts on colony growth in social insects due to effects on brood (Porter 1988; Asano \& Cassill 2012). Changes in nest temperature of only $3-5^{\circ} \mathrm{C}$ from optimum can slow or halt brood development even when colonies have sufficient resources for growth (Porter 1988; Kipyatkov \& Lopatina 2015). To compensate, some species build mounds or other structures that collect solar heat or generate heat through compost decomposition (Rosengren et al. 1987; Penick \& Tschinkel 2008; Vogt et al. 2008). More generally, species respond behaviorally by relocating brood throughout the day or season to track preferred temperatures and moisture levels (Potts et al. 1984; Roces \& Núñez 1989; Penick \& Tschinkel 2008; $\mathrm{Xu}$ et al. 2009). Workers of fire ants and honey bees can discriminate changes in nest temperature as little as $0.25-2^{\circ} \mathrm{C}$ (Winston 1987; Porter \& Tschinkel 1993), and this ability may allow species to respond to extremely narrow temperature gradients. While the majority of studies on brood thermoregulation have focused on species that build large or specialized nests, brood thermoregulation is likely important more generally, even for species that live in relatively small nests.

The same benefits that nests provide to the animals that inhabit them-controlled temperature and moisture conditions - also make nests ideal habitats for microbes and potential pathogens. Soil and leaf litter contain some of the highest diversities of microbes on record (Torsvik \& Øvreås 2002) and pose a challenge to species that build nests in these habitats. Social insects are particularly at risk to pathogen exposure due to their dense living conditions and high interaction rates (Fefferman \& Traniello 2009). Ants developed specialized glands early in their evolution to inhibit microbial growth (Yek \& Mueller 2011), and species produce a variety of antimicrobial compounds (Schluns \& Crozier 2009). However, the production of antimicrobials is metabolically expensive and may incur significant costs for small or newly founded colonies (Poulsen et al. 2002). An alternative approach is for colonies to contain or avoid microbes inside their nests by designating certain areas as refuse chambers (Bot et al. 2001) or 'toilets' (Czaczkes et al. 2015) as well as avoiding areas where microbes are abundant or difficult to control. In this way, microbes may influence behavior and nest organization in addition to microclimate factors.

Here we studied how colonies of the acorn ant Temnothorax curvispinosus responded to variation in temperature, moisture, and microbial growth inside their nests. T. curvispinosus is among the most common ant species in eastern forests of North America (Pearse 1946; Stuble et al. 2013), and their colonies nest inside fallen acorns or hickory nuts on the forest floor (Fig. 1). While large colonies may fill an acorn entirely, small- to medium-sized colonies generally inhabit only a small portion of an acorn. Colonies may have room to adjust their position in response to microclimate or microbial factors that could differ between the tops and bottom halves of an acorn. The top is more exposed to solar heating and relatively dry, while the bottom is often embedded in moist soil, which favors the growth of microbes (Clark 1967). Working with colonies in the laboratory, we tested three main hypotheses for how colonies would respond to different nest conditions:

1 Moisture preference-Colonies will prefer moist regions of the nest (bottom) over dry regions to prevent desiccation.

2 Temperature preference-Colonies will prefer heated regions of the nest (top) over cool regions to stimulate brood production.
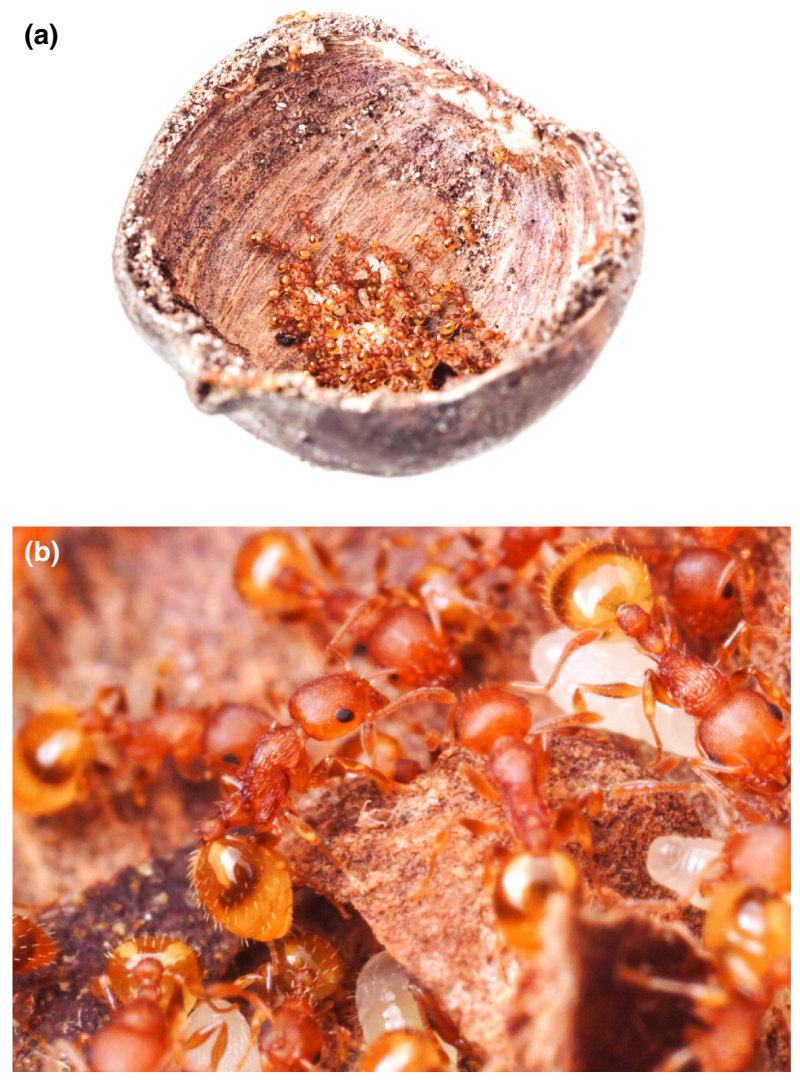

Fig. 1: (a) Temnothorax curvispinosus colony inhabiting the bottom half of an acorn nest (top half removed); (b) close-up view of ants inside the nest (Photographs by Lauren Nichols). 
3 Microbe avoidance-Colonies will avoid regions of the nest that favor microbial growth (bottom) to prevent pathogen exposure.

We evaluated each hypothesis independently to compare which factor(s) had the strongest impact on nest organization in T. curvispinosus. We then discuss how our results may apply to other nest-inhabiting species.

\section{Methods}

\section{Study Species and Laboratory Conditions}

We collected whole colonies of Temnothorax curvispinosus from populations near Raleigh, NC, USA $\left(35.7611^{\circ} \mathrm{N}, 78.6836^{\circ} \mathrm{W}\right.$, elevation $\left.107 \mathrm{~m}\right)$, between spring 2013 and fall 2014. All colonies contained at least one queen (some contained up to 6 queens, which is in line with previous accounts of polygyny in this species (Alloway et al. 1982)) and 17-174 workers. Colonies of this species nest inside fallen acorns or hickory nuts, and we provided each colony with a hollowed acorn to serve as a nest chamber in the lab by splitting an acorn (Quercus spp., $\sim 16 \times 16 \mathrm{~mm}$ ) along its longitudinal axis and removing the nut to leave an empty interior space (Fig. 1). A small hole ( $\sim 2 \mathrm{~mm}$ diameter) was left open between the top and bottom halves of the acorn to serve as a nest entrance, and both halves were held together with a small rubber band (15 mm diameter). We fed colonies ad libitum with a combination of frozen mealworms (Zophobas morio), vials containing 20\% sucrose solution, and an artificial diet designed specifically for ants (Bhatkar \& Whitcomb 1970).

\section{Moisture Preference Experiment}

To test whether T. curvispinosus colonies displayed a moisture preference, we allowed colonies to move into acorn nests after either the top or bottom half had been soaked in water, while the other side was left dry. Acorn halves were soaked in water for $30 \mathrm{~min}$, and the moistened half was then covered with paraffin film on the outside to prevent drying during a trial. Colonies generally moved into their new acorns within one hour, and we counted workers and brood (larvae and pupae) on either half after $6 \mathrm{~h}$ or $12 \mathrm{~h}$ from the time new nests were provided (results were similar for both time points, so we combined data from all trials in our final analyses). As a measure of nest moisture, we weighed acorn halves before and after soaking in water as well as at the end of our trials. Soaked acorns increased in weight by an average of $41 \%(0.35 \mathrm{~g})$ due to water gain after soaking, and they maintained an increase in weight of $33 \%$ and $27 \%$ during trials of $6 \mathrm{~h}$ and $12 \mathrm{~h}$, respectively. The weight of dry acorn halves remained close to the same before and after the experiment (an average increase in weight of $5 \%$ ), which indicates that they remained relatively dry throughout our trials. We used 13 colonies in trials with the top half of the acorn moistened and 13 colonies in trials with the bottom half of the acorn moistened.

\section{Temperature Preference Experiment}

To test whether T. curvispinosus colonies displayed a temperature preference, we heated acorns from above to simulate warming by the sun. Colonies were allowed to move into dry acorns, which were then transferred to a cooling block (VWR, Radnor, PA, USA). The bottom half of the acorn was imbedded in soft clay cooled to $18^{\circ} \mathrm{C}$ (monitored with a thermometer throughout the experiment), while the top half was heated using a heat lamp (red light, 75 watts) positioned approximately $0.5 \mathrm{~m}$ above the acorn. Small strips of paper were placed on top of the clay to create an insulating layer akin to leaf litter. This leafy analogue did not cover the top of the acorn, which remained under direct light. Colonies were heated for $2 \mathrm{~h}$, at which time nests were opened to count workers and brood (larvae and pupae) on either half. Temperature readings using an infrared thermometer aimed at the top of the acorn registered $36 \pm 1{ }^{\circ} \mathrm{C}$ at the time colonies were opened. For the control group, all steps and conditions were replicated except the cooling block and heat lamp were not turned on. We used 17 colonies in heated trials and 17 colonies in unheated controls.

\section{Microbe Avoidance Experiment}

In addition to moisture and temperature, we tested whether T. curvispinosus colonies adjusted their position in response to microbial growth on the bottom of their nests. We cultured microbes from an old acorn nest that had visible cultures of moldy fungi. This acorn nest was initially constructed in the laboratory from a dried acorn, and the cultures growing on it likely came from the ants themselves or from microbes generally present in the laboratory environment. We washed the inside of this acorn with $100 \mu \mathrm{l}$ sterile DI water and spread the washing on a Petri plate of potato dextrose agar. Plates were incubated at $37^{\circ} \mathrm{C}$ until fungal growth covered most of the plate, and then the plates were stored at $4{ }^{\circ} \mathrm{C}$ before inoculating new nests. The plates featured a mix of several 
morphologically distinct cultures of filamentous fungi. Because we were not interested in the response of T. curvispinosus to any one specific microbe species, we inoculated new nests with a mix of cultures. We used a sterile spatula to take $\sim 2 \mathrm{~mm}^{2}$ of fungal mycelia from three locations of the Petri plate that featured distinct microbe cultures. We transferred these cultures to an acorn half that was then kept moist at $25^{\circ} \mathrm{C}$ for $1-2 \mathrm{wk}$ to give time for microbes to grow (all acorns were inoculated with the same three microbe cultures). The acorn was then rejoined with its missing half, and colonies were given $6 \mathrm{~h}$ to move into the nest. Nests were opened after $6 \mathrm{~h}$ to count workers and brood (larvae and pupae) on either half. For the control group, all steps and conditions were replicated except colonies were given acorns with two clean halves. We conducted trials with 13 colonies in acorns inoculated with microbes and 17 colonies in clean acorns as a control.

Previous observations suggested colonies moved to the top of their acorn nests in the laboratory over time. We predicted that colonies might move to the top of the acorn in response to microbial growth on the bottom half of their acorn. In nature, acorns are generally imbedded in moist soil, which would likely promote the growth of mold and other microbes. Therefore, we simulated this condition in the laboratory by providing seven colonies with new acorn nests and embedded the bottom of these acorns in moist sand. We opened acorns once per week to assess the position of the colony inside the nest for a period that lasted up to 34 wk. We noted when more than $90 \%$ of the colony was found on the top half of the acorn for at least two consecutive weeks (a clear indication that colonies had relocated to the top of the acorn). After this time, we flipped the acorns and checked the colony position 27 days after the acorns were flipped. If colonies initially avoided the bottom half of an acorn due to the growth of microbes, then we predicted colonies would continue to avoid this half when their acorn was flipped (i.e., they would stay on the 'clean' half of the acorn rather than move back to the top). On the other hand, if colonies had developed a general preference for the top of the acorn that was not due to avoidance of microbes, then we predicted colonies would move back to the top of the acorn after it was reversed (i.e., they would shift from the 'clean' half of the acorn to the potentially 'microbe-covered' top).

\section{Statistical Analyses}

We tested whether colonies changed their location inside their nests using the Mann-Whitney $U$-test to compare the proportion of workers and brood on the top of their nests between treatment and control colonies. The Mann-Whitney $U$-test is a more conservative, nonparametric test (it does not require the assumption of normally distributed data). For all experiments, colonies were only tested once for each treatment to maintain independence and to avoid pseudoreplication. Statistical tests were conducted using GraphPad Prism 6 (GraphPad Software, Inc. 2015) statistical software.

\section{Results}

\section{Moisture Preference}

Colonies of Temnothorax curvispinosus showed no moisture preference (Fig. 2a). There was no difference in the position of workers (Mann-Whitney $U$-test, $\mathrm{n}=13, U=77.5, \mathrm{p}=0.71$ ) or brood (Mann-Whitney $U$-test, $\mathrm{n}=13, U=84.5, \mathrm{p}=0.99)$ inside the nest when either the top half or bottom half of the acorn was moistened. Instead, all colonies moved to the bottom of the nest regardless of moisture treatment.

\section{Temperature Preference}

Colonies showed a preference for the top half of the acorn when it was heated to simulate warming by the sun (Fig. 2b). The proportion of brood on the top half of heated acorns was significantly higher compared with unheated controls (Mann-Whitney $U$-test, $\mathrm{n}=17, U=87, \mathrm{p}=0.0132$ ), and the proportion of workers was also higher but did not differ significantly from unheated controls (Mann-Whitney $U$-test, $\mathrm{n}=17, U=118, \mathrm{p}=0.37$ ). The movement of colonies to the top half of the acorn indicated a preference for warmer temperatures, which would increase the rate of brood development. In contrast, nearly all workers $(91 \%)$ and brood $(97 \%)$ were found on the bottom half of nests in unheated controls (Fig. 2b).

\section{Microbe Avoidance}

When the bottom half of an acorn was inoculated with microbe cultures, a significant portion of colonies shifted to the top half of the acorn after $6 \mathrm{~h}$ (Fig. 2c). The proportion of both workers (MannWhitney $U$-test, $\mathrm{n}_{\text {control }}=17, \mathrm{n}_{\text {microbes }}=13, U=49$, $\mathrm{p}=0.0090)$ and brood (Mann-Whitney $U$-test, $\mathrm{n}_{\text {con- }}$ trol $=17$, nmicrobes $=11, U=46, \mathrm{p}=0.0147$ ) was higher on the top half of inoculated acorns compared with non-inoculated controls. In contrast, $73 \%$ of workers and $93 \%$ of larvae and pupae were found on the bottom half of control acorns. 

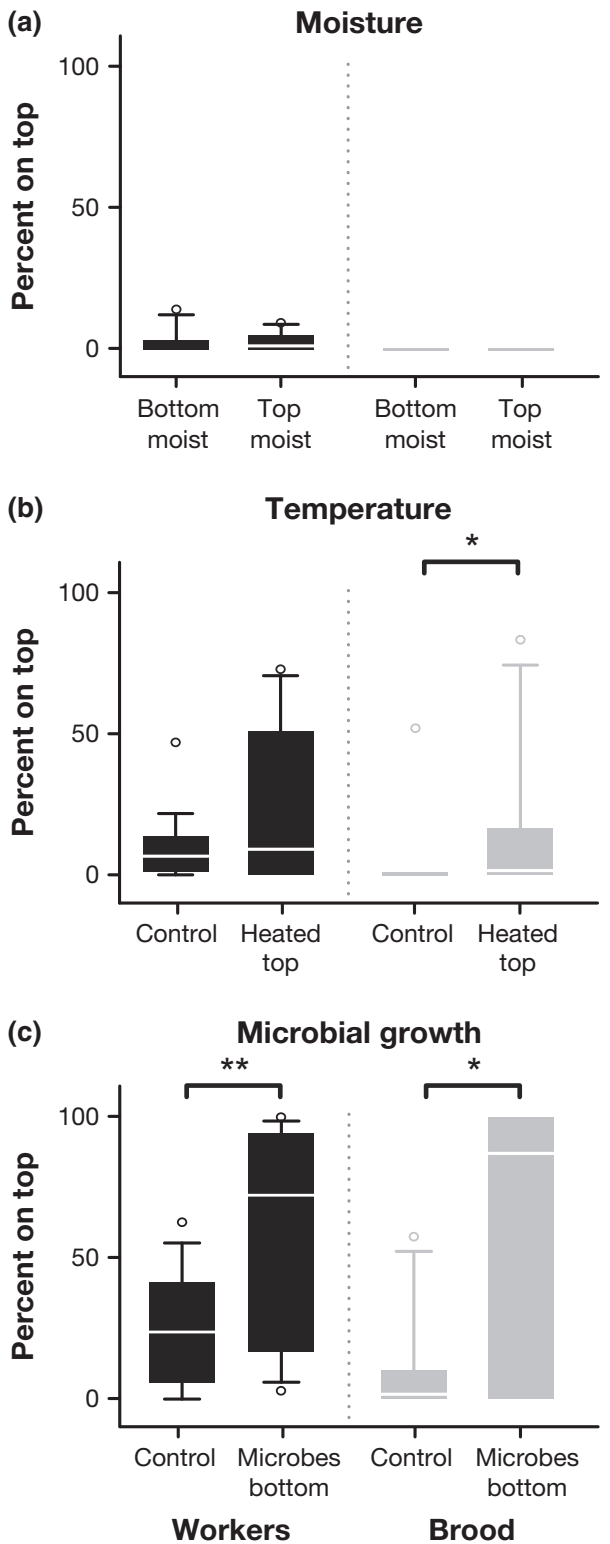

Fig. 2: Location of workers and brood inside acorn nests in response to (a) moisture, (b) temperature, and (c) microbial treatments represented by boxplots showing median, 25-75\%, and non-outlier range (open circles indicate outliers). Moisture had no effect on colony location with all colony members located at the bottom of the nest regardless of treatment $(n=13)$. Heating the top of the nest resulted in a greater proportion of the colony's brood on the top half of the nest compared with unheated controls $(n=17)$. Microbial growth on the bottom of the nest resulted in a higher proportion of both workers $\left(n_{\text {control }}=17, n_{\text {microbes }}=13\right)$ and brood $\left(n_{\text {control }}=17, n_{\text {microbes }}=11\right)$ on the top half of the nest compared with untreated controls. Asterisks indicate significant differences $(* p<0.05, * * p<0.01)$.

We monitored colonies in the laboratory for 7 $34 \mathrm{wk}$ to see how their position inside new nests changed over time as microbes were given time to grow. All colonies spent the first $1-5$ wk predominantly on the bottom half of the acorn. Colonies began to shift to the top of the acorn by week 7 (indicated when more than $90 \%$ of the colony was found on the top half), and over $50 \%$ of colonies had shifted to the top of the acorn by week 16 (Fig. 3). In addition, all nests had visible signs of microbial growth by the end of each trial. When a colony was consistently found on the top of the acorn (more than $90 \%$ of the colony on the top half of the acorn for two consecutive weeks), we flipped the acorn to test whether colonies would stay on the bottom-indicating potential microbe avoidance-or whether colonies would return to the top where they were most recently located. Out of seven colonies, only one moved back to the top within $1 \mathrm{wk}$, while all other colonies remained on the bottom half, consistent with the microbe avoidance hypothesis.

\section{Discussion}

Nests provide shelter and protection for ant colonies from environmental extremes, but a nests' ultimate utility depends on how colonies use behavior to track optimal conditions and prevent pathogen exposure. We found that colonies of the ant Temnothorax curvispinosus shifted their location inside their nests in response to two primary factors: temperature and microbial growth. When colonies were heated from the top to simulate warming by the sun, workers moved brood to the warm, top half of the nest, which would stimulate brood development. In contrast,

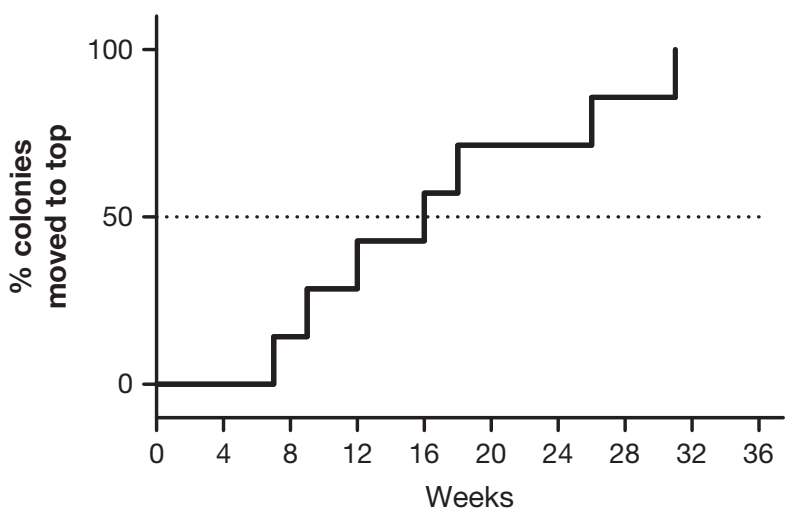

Fig. 3: Colony transition from the bottom to the top half of laboratory nests over time. All colonies were located on the bottom half of their acorns for the first $6 \mathrm{wk}$, but colonies transitioned to the top half over time (counted when more than $90 \%$ of a colony was found on the top half of their acorn). This transition was consistent with the microbial avoidance hypothesis, which suggests colonies move to the top of the acorn to avoid microbes that grow on the bottom over time. 
colonies showed no response to differences in moisture inside their nests and generally moved to the bottom of the acorn regardless of moisture treatment, the strongest factor that influenced colony position inside the nest was the presence of microbes-colonies avoided the bottom halves of their nests when they were inoculated with microbes, and colonies also tended to shift to the top halves of their acorns in new nests after microbes had time to grow. While ants and other social insects produce antimicrobial compounds, avoiding regions of the nest where microbes are abundant or difficult to control may be an additional line of defense to limit exposure to potential pathogens.

Unlike their responses to temperature and microbial abundance, T. curvispinosus colonies did not change their location in response to moisture. Low moisture environments can provide a challenge for small insects that are susceptible to desiccation, but ant species vary in their ability to tolerate dry conditions. While species that nest in soil generally require high humidity, ant species that nest in drier habitats, such as trees or woody material above ground, are generally more tolerant of dry conditions (Hood \& Tschinkel 1990). Because T. curvispinosus colonies nest inside acorns that can dry out when humidity is low, T. curvispinosus colonies may be more tolerant of dry conditions. In fact, one closely related speciesT. wheeleri-is among the most desiccation-resistant of all ant species that have been studied (Hood $\delta$ Tschinkel 1990). High tolerance for dry conditions may explain why T. curvispinosus colonies showed no preference for moist or dry regions of their nests, although moisture inside the nest is likely important for other ant species (Xu et al. 2009).

Colonies of T. curvispinosus moved to the top of their acorns when they were heated from above to simulate warming by the sun. Warm temperatures stimulate brood development (Porter 1988; Kipyatkov \& Lopatina 2015), and T. curvispinosus has relatively high thermal requirements for growth compared with other co-occurring species (Pelini et al. 2012; Diamond et al. 2013). When acorns were heated in the laboratory, colonies brought brood to the top half of the acorn where warm temperatures would increase brood development rate. What was remarkable in T. curvispinosus is that they were able to take advantage of a temperature gradient inside the confines of a tiny acorn. Other ants adjust the position of brood to take advantage of solar heating (Penick $\delta$ Tschinkel 2008; Anderson \& Munger 2009), but these species often build large nests as well as construct mounds that act as solar collectors. If T. curvispinosus colonies actively track temperature inside nests as small as an acorn, then thermoregulatory behaviors are likely important for most if not all ant species, even those that live in relatively small nests. The degree to which solar heating occurs in T. cusrvispinosus nests in the field, however, requires further study. If acorns are fully exposed to the sun, then heating is likely to occur, but this may not apply in areas where acorns are in the shade or covered by leaf litter. This could explain why colonies responded more strongly to the presence of microbes in their nests than their response to nest temperature.

While temperature and moisture are the primary factors thought to affect nest organization in social insects, the strongest factor for T. curvispinosus was the presence of microbes. Colonies of T. curvispinosus avoided areas of the nest where visible microbes were already present, and colonies in untreated acorns moved to the top of the nest over several weeks as mold or other microbes had time to grow. The bottom half of an acorn in the field is generally embedded in moist soil-which favors the growth of microbes (Barros et al. 1995) - and we created similar conditions in the lab by embedding acorns in moist sand. Colonies presumably moved to the dry, upper halves of their acorns because microbes there were easier to control. The ability of social insects to cope with microbial communities in their environment and to mitigate pathogen exposure is key to their ecological success (Fefferman \& Traniello 2009). Compared with solitary species, social insects invest more heavily in antimicrobial compounds to kill or slow microbial growth (Stow et al. 2007; Hoggard et al. 2011). Our results suggest further that colonies aggregate in areas of the nest where microbes are less abundant or potentially easier to control, although there is evidence from others that ant colonies prefer nests with low levels of pathogens present that could facilitate immune priming (Konrad et al. 2012; Pontieri et al. 2014). Nevertheless, avoiding regions of the nest that favor microbial growth may allow smaller colonies to conserve resources that would otherwise be devoted to the production of antimicrobials. In contrast, large colonies may be able to invest more heavily in antimicrobial production due to lower costs per capita.

How common are behaviors used by social insect species to avoid pathogen exposure inside their nests? One clue is the presence of specialized larval hairs found in a variety of ant species that allow workers to hang larvae from the ceiling of the nest rather than leaving larvae on the floor (Wheeler \& Wheeler 1976; Penick et al. 2012). Our findings for T. curvispinosus suggest that the ceiling of the nest may be more 
sanitary than the floor due to differences in moisture levels. Hanging larvae from the ceiling may therefore elevate larvae above areas where microbes are abundant or difficult to control. In T. curvispinosus, larvae possess 'anchor-tipped' hairs that serve this purpose, and anchor-tipped hairs are found on larvae from at least 22 ant genera within the subfamily Myrmicinae (Penick et al. 2012). In other ant genera, 'singlehooked' hairs and 'sticky doorknobs' may serve a similar purpose (Peeters \& Hölldobler 1992; Penick et al. 2012). This suggests that hanging larvae from the ceiling of the nest is a common practice in ants that may help avoid pathogen exposure. In addition, hanging larvae from the ceiling of the nest could serve a thermoregulatory purpose by placing larvae in closer contact with nest surfaces heated by the sun. Both factors seem to be important for T. curvispinosus, and future studies on social insects should consider both microclimate and pathogen exposure as factors that influence nest organization.

\section{Acknowledgements}

We received help collecting and maintaining colonies from Mary Vincent and Matt Farrell. Some of our acorns were unwittingly supplied by Kurt Karlik. We would also like to thank the Silverman lab for providing space to conduct our moisture trials, and Lauren Nichols, who took photographs of our Temnothorax colonies. This work was supported by a National Science Foundation Dimensions of Biodiversity grant (NSF-1 136703) awarded to RRD and a North Carolina State University undergraduate research award to JK. The authors have no conflicts of interest to declare.

\section{Literature Cited}

Alloway, T. M., Buschinger, A., Talbot, M., Stuart, R. \& Thomas, C. 1982: Polygyny and polydomy in three North American species of the ant genus Leptothorax Mayr (Hymenoptera: Formicidae). Psyche 89, 249274.

Anderson, K. E. \& Munger, J. C. 2009: Effect of temperature on brood relocation in Pogonomyrmex salinus (Hymenopteria: Formicidae). West. N. Am. Nat. 63, 122 $-128$.

Asano, E. \& Cassill, D. L. 2012: Modeling temperaturemediated fluctuation in colony size in the fire ant, Solenopsis invicta. J. Theor. Biol. 305, 70-77.

Barros, N., Gomez-Orellana, I., Feijóo, S. \& Balsa, R. 1995: The effect of soil moisture on soil microbial activity studied by microcalorimetry. Thermochim. Acta 249, $161-168$.
Bhatkar, A. \& Whitcomb, W. H. 1970: Artificial diet for rearing various species of ants. Fla. Entomol. 53, 229232.

Bot, A., Currie, C., Hart, A. \& Boomsma, J. J. 2001: Waste management in leaf-cutting ants. Ethol. Ecol. Evol. 13, 225-237.

Clark, F. E. 1967: Bacteria in soil. In: Soil Biology. (Burges, N. A., Raw, F., eds). Academic Press, New York, NY, pp. 15-49.

Czaczkes, T. J., Heinze, J. \& Ruther, J. 2015: Nest etiquette -where ants go when nature calls. PLoS ONE 10 e0118376.

Diamond, S. E., Penick, C. A., Pelini, S. L., Ellison, A. M., Gotelli, N. J., Sanders, N. J. \& Dunn, R. R. 2013: Using physiology to predict the responses of ants to climatic warming. Integr. Comp. Biol. 53, 965-974.

Fefferman, N. H. \& Traniello, J. F. A. 2009: Social insects as models in epidemiology: establishing the foundation for an interdisciplinary approach to disease and sociality. In: Organization of Insect Societies: From Genome to Sociocomplexity. (Gadau, J. \& Fewell, J., eds). Harvard Univ. Press, Cambridge, MA, pp. 545-571.

Hoggard, S. J., Wilson, P. D., Beattie, A. J. \& Stow, A. J. 2011: Social complexity and nesting habits are factors in the evolution of antimicrobial defences in wasps. PLoS ONE 6, e21763.

Hood, W. G. \& Tschinkel, W. R. 1990: Desiccation resistance in arboreal and terrestrial ants. Physiol. Entomol. 15, 23-35.

Jones, J. C. \& Oldroyd, B. P. 2006: Nest thermoregulation in social insects. Adv. Insect Phys. 33, 153-191.

Kinlaw, A. 1999: A review of burrowing by semi-fossorial vertebrates in arid environments. J. Arid Environ. 41, $127-145$.

Kipyatkov, V. E. \& Lopatina, E. B. 2015: Comparative study of thermal reaction norms for development in ants. Entomol. Sci. 18, 174-192.

Konrad, M., Vyleta, M. L., Theis, F. J., Stock, M., Tragust, S., Klatt, M., Drescher, V., Marr, C., Ugelvig, L. V. \& Cremer, S. 2012: Social transfer of pathogenic fungus promotes active immunisation in ant colonies. PLoS Biol. 10, e1001300.

Korb, J. 2003: Thermoregulation and ventilation of termite mounds. Naturwissenschaften 90, 212-219.

Mezger, D. \& Pfeiffer, M. 2010: Is nest temperature an important factor for niche partitioning by leaf-litter ants (Hymenoptera: Formicidae) in Bornean rain forests? J. Trop. Ecol. 26, 445-455.

Pearse, A. 1946: Observations on the microfauna of the Duke forest. Ecol. Monogr. 16, 127-150.

Peeters, C. \& Hölldobler, B. 1992: Notes on the morphology of the sticky "doorknobs" of larvae in an Australian Hypoponera sp. (Formicidae; Ponerinae). Psyche 99, 23-30. 
Pelini, S. L., Diamond, S. E., MacLean, H., Ellison, A. M., Gotelli, N. J., Sanders, N. J. \& Dunn, R. R. 2012: Common garden experiments reveal uncommon responses across temperatures, locations, and species of ants. Ecol. Evol. 2, 3009-3015.

Penick, C. A. \& Tschinkel, W. R. 2008: Thermoregulatory brood transport in the fire ant, Solenopsis invicta. Insectes Soc. 55, 176-182.

Penick, C. A., Copple, R. N., Mendez, R. A. \& Smith, A. A. 2012: The role of anchor-tipped larval hairs in the organization of ant colonies. PLoS ONE 7, e41595.

Pontieri, L., Vojvodic, S., Graham, R., Pedersen, J. S. \& Linksvayer, T. A. 2014: Ant colonies prefer infected over uninfected nest sites. PLOS ONE 9, el11961.

Porter, S. D. 1988: Impact of temperature on colony growth and developmental rates of the ant, Solenopsis invicta. J. Insect Physiol. 34, 1127-1133.

Porter, S. D. \& Tschinkel, W. R. 1993: Fire ant thermal preferences: behavioral control of growth and metabolism. Behav. Ecol. Sociobiol. 32, 321-329.

Potts, L., Francke, O. \& Cokendolpher, J. 1984: Humidity preferences of four species of fire ants (Hymenoptera: Formicidae: Solenopsis). Insectes Soc. 31, 335-340.

Poulsen, M., Bot, A. N., Nielsen, M. G. \& Boomsma, J. J. 2002: Experimental evidence for the costs and hygienic significance of the antibiotic metapleural gland secretion in leaf-cutting ants. Behav. Ecol. Sociobiol. 52, 151-157.

Roces, F. \& Núñez, J. A. 1989: Brood translocation and circadian variation of temperature preference in the ant Camponotus mus. Oecologia 81, 33-37.

Rosengren, R., Fortelius, W., Lindström, K. \& Luther, A. 1987: Phenology and causation of nest heating and thermoregulation in red wood ants of the Formica rufa group studied in coniferous forest habitats in southern Finland. Ann. Zool. Fennici. 24, 147-155.
Schluns, H. \& Crozier, R. H. 2009: Molecular and chemical immune defenses in ants (Hymenoptera: Formicidae). Myrmecol. News 12, 237-249.

Schwarzkopf, L. \& Alford, R. 1996: Desiccation and shelter-site use in a tropical amphibian: comparing toads with physical models. Funct. Ecol. 10, 193-200.

Stow, A., Briscoe, D., Gillings, M., Holley, M., Smith, S., Leys, R., Silberbauer, T., Turnbull, C. \& Beattie, A. 2007: Antimicrobial defences increase with sociality in bees. Biol. Lett. 3, 422-424.

Stuble, K. L., Rodriguez-Cabal, M. A., McCormick, G. L., Jurić, I., Dunn, R. R. \& Sanders, N. J. 2013: Tradeoffs, competition, and coexistence in eastern deciduous forest ant communities. Oecologia 171, 981—992.

Torsvik, V. \& Øvreås, L. 2002: Microbial diversity and function in soil: from genes to ecosystems. Curr. Opin. Microbiol. 5, 240-245.

Turner, J. S. 2009: The Extended Organism: The Physiology of Animal-Built Structures. Harvard Univ. Press, Cambridge, MA.

Vogt, J. T., Wallet, B. \& Coy, S. 2008: Dynamic thermal structure of imported fire ant mounds. J. Insect Sci. 8, 31 .

Wheeler, G. C. \& Wheeler, J. 1976: Ant Larvae: Review and Synthesis. The Entomological Society of Washington, Washington, DC.

Wiebe, K. L. 2001: Microclimate of tree cavity nests: is it important for reproductive success in Northern Flickers? Auk 118, 412-421.

Winston, M. L. 1987: The Biology of the Honey Bee. Harvard Univ. Press, Cambridge, MA.

Xu, Y., Zeng, L., Lu, Y. \& Liang, G. 2009: Effect of soil humidity on the survival of Solenopsis invicta Buren workers. Insectes Soc. 56, 367-373.

Yek, S. H. \& Mueller, U. G. 2011: The metapleural gland of ants. Biol. Rev. 86, 774-791. 